\title{
Improved outcomes with minimally invasive pancreaticoduodenectomy in patients with dilated pancreatic ducts: a prospective study
}

\author{
Heidy $\operatorname{Cos}^{1,4} \cdot$ Michael T. LeCompte ${ }^{2}$. Sanket Srinivasa ${ }^{1}$ Jorge Zarate Rodriguez ${ }^{1,4}$. Cheryl A. Woolsey ${ }^{1,4}$. \\ Gregory Williams $^{1,4}$. Siddarth Patel ${ }^{4}$. Adeel Khan ${ }^{1,3,4}$. Ryan C. Fields ${ }^{1,3,4} \cdot$ Maria B. Majella Doyle M $^{1,3,4}$. \\ William C. Chapman ${ }^{1,3,4}$. Steven M. Strasberg ${ }^{1,3,4}$. William G. Hawkins ${ }^{1,3,4}$. Chet W. Hammill ${ }^{1,3,4}$. \\ Dominic E. Sanford ${ }^{1,3,4}$
}

Received: 17 March 2021 / Accepted: 14 June 2021 / Published online: 7 July 2021

(c) The Author(s), under exclusive licence to Springer Science+Business Media, LLC, part of Springer Nature 2021

\begin{abstract}
Background Little is known about what factors predict better outcomes for patients who undergo minimally invasive pancreaticoduodenectomy (MIPD) versus open pancreaticoduodenectomy (OPD). We hypothesized that patients with dilated pancreatic ducts have improved postoperative outcomes with MIPD compared to OPD.

Methods All patients undergoing pancreaticoduodenectomy were prospectively followed over a time period of 47 months, and perioperative and pathologic covariates and outcomes were compared. Ideal outcome after PD was defined as follows: (1) no complications, (2) postoperative length of stay $<7$ days, and (3) negative (R0) margins on pathology. Patients with dilated pancreatic ducts $(\geq 3 \mathrm{~mm})$ who underwent MIPD were 1:3 propensity score-matched to patients with dilated ducts who underwent OPD and outcomes compared. Likewise, patients with non-dilated pancreatic ducts $(<3 \mathrm{~mm})$ who underwent MIPD were 1:3 propensity score-matched to patients with non-dilated ducts who underwent OPD and outcomes were compared.

Results 371 patients underwent PD-74 (19.9\%) MIPD and 297 (80.1\%) underwent OPD. Overall, patients who underwent MIPD had significantly less intraoperative blood loss. After 1:3 propensity score matching, patients with dilated pancreatic ducts who underwent MIPD $(n=45)$ had significantly lower overall complication and 90-day readmission rates compared to matched OPD patients $(n=135)$ with dilated ducts. Patients with dilated duct who underwent MIPD were more likely to have an ideal outcome than patients with OPD ( $29 \mathrm{vs} 15 \%, p=0.035)$. There were no significant differences in postoperative outcomes among propensity score-matched patients with non-dilated pancreatic ducts who underwent MIPD $(n=29)$ compared to matched patients undergoing OPD $(n=87)$ with non-dilated ducts.

Conclusions MIPD is safe with comparable perioperative outcomes to OPD. Patients with pancreatic ducts $\geq 3 \mathrm{~mm}$ appear to derive the most benefit from MIPD in terms of fewer complications, lower readmission rates, and higher likelihood of ideal outcome.
\end{abstract}

Keywords Minimally invasive pancreatoduodenectomy $\cdot$ Outcomes $\cdot$ Ideal outcome $\cdot$ Robotic $\cdot$ Laparoscopic

Minimally invasive pancreaticoduodenectomy (MIPD), including robotic-assisted and totally laparoscopic, is a

Dominic E. Sanford

sanfordd@wustl.edu

1 Division of Hepatobiliary, Pancreatic, and Gastrointestinal Surgery, Department of Surgery, Barnes-Jewish Hospital, Washington University School of Medicine, 660 South Euclid Avenue, Campus Box 8109, Saint Louis, MO 63110, USA complex procedure with a steep learning curve $[1,2]$. The benefits of MIPD over open pancreaticoduodenectomy 
(OPD) are controversial. Goals of using a minimally invasive approach in pancreaticoduodenectomy (PD) include reducing postoperative complications and the time needed for recovery while maintaining quality oncologic outcomes $[3,4]$. While there is prospective evidence suggesting MIPD can accomplish these goals, there are also reports of worse outcomes with MIPD [5-7]. Uncovering the advantages and disadvantages of MIPD will be critical to determining what role (if any) this procedure should play in the care of patients with periampullary pathology. Further prospective study of MIPD at high-volume pancreatectomy centers, where MIPD can be performed safely, with low mortality, is needed [8].

Postoperative pancreatic fistula is the major driver of morbidity and mortality after pancreaticoduodenectomy $[9,10]$. Therefore, patients who develop pancreatic fistulas after PD may be less likely to experience a benefit from a minimally invasive approach compared to patients without pancreatic fistulas. However, in most modern series, the majority of patients undergoing PD do not experience a pancreatic fistula postoperatively [11]. The risk factors for pancreatic fistula after PD are well described, with small pancreatic ducts and soft pancreatic gland texture being the primary predisposing factors [11-14]. Of these, pancreatic duct size can be objectively measured preoperatively with commonly used radiologic and ultrasound imaging modalities. Understanding preoperatively which patients (if any) benefit from MIPD could be useful, as starting an MIPD program is not only costly and resource intensive but is also associated with a steep learning curve [2]. Such knowledge, may allow surgeons to better optimize preoperative patient selection, which might be especially valuable during one's early experience with MIPD.

In 2016, we began performing MIPD at our institution as a part of a prospective registry in order to examine its impact on postoperative patient outcomes. In this study, we compare the outcomes of patients undergoing MIPD to those undergoing OPD. We sought to determine if MIPD benefits patients with regard to reduced postoperative complications compared to OPD. We hypothesized that patients with dilated pancreatic ducts (i.e., at lower risk of pancreatic fistula) who undergo MIPD experience fewer postoperative complications.

\section{Materials and methods}

MIPD was first performed at the authors' institution in April 2016. All consecutive patients between April 2016 and February 2020 undergoing PD at Barnes-Jewish Hospital were prospectively followed for 90 days postoperatively in a complications database. In March of 2020 institutional policy limited minimally invasive cases due to uncertainty regarding COVID-19 exposure with insufflation and de-sufflation of the abdomen. Patient demographic, perioperative, and pathologic data were also collected. All complications were prospectively collected and graded for severity by a trained physician assistant. Results were presented at a weekly surgical conference at which time the type and grading of complications were confirmed or adjusted [15]. Whenever feasible, NSQIP definitions of postoperative complications were used. Complication severity was judged using the Modified Accordion Grading System (MAGS) [16]. MAGS is a validated severity grading system based primarily on the type and morbidity of interventions needed to treat a complication. It was derived from a severity grading system first published in 1992 by Clavien et al. [17] and modified in the course of validation and hence the term "modified" [16]. There are 6 grades of complications. The highest-grade complication in MAGS is death (grade 6). Patients without complications are graded as zero. "Severe complications" are those that are grade 3 or higher.

Any postoperative blood transfusion from 0 to 90 days was considered to be a grade 2 complication.

Drain fluid amylase levels were measured on all patients with drains, and pancreatic fistula was defined as drain fluid having an amylase level greater than 3 times the upper limit of normal serum value on postoperative day 3 onward. Clinically relevant pancreatic fistula was defined as grade B/C fistulas according to the International Study Group of Pancreatic Fistula (ISGPF) guidelines [18]. Pancreatic duct size was considered dilated if $3 \mathrm{~mm}$ or greater and non-dilated if less than $3 \mathrm{~mm}$ as reported by surgeons at the time of operation. This cutoff was chosen based on the authors' previous work using the smallest pancreatic duct category in the National Surgical Quality Improvement Program (NSQIP) pancreatectomy database of less than $3 \mathrm{~mm}$. The specific complication "bleeding/anemia" refers to a documented postoperative bleeding event or postoperative anemia requiring blood transfusion at any time in the postoperative period up to 90 days. We typically use a hemoglobin of less than $7 \mathrm{~g} / \mathrm{dl}$ as our threshold for blood transfusion. Ideal outcome was created as a composite outcome to examine the impact of MIPD vs OPD on perioperative results. Ideal outcome was defined as patients having all three of the following: (1) no MAGS complications (including no death and no readmission); (2) a postoperative length of hospital stay equal to or less than the overall median length of stay (i.e., 7 days); and (3) negative (R0) pathologic margins (i.e., tumor $>1 \mathrm{~mm}$ from margin). Pathologic margins were considered for any neoplasms including pancreatic ductal adenocarcinoma (PDAC) and neuroendocrine tumors.

MIPD was defined as totally laparoscopic, roboticassisted, or laparoscopic/robotic converted to open. All surgeons who performed MIPD performed both totally laparoscopic as well as robotic-assisted procedures, with each MIPD surgeon performing at least 3 of each. Four of the 
8 surgeons were performing MIPD and one of the authors (DES) was present for 55\% of MIPDs as primary surgeon or co-surgeon/assistant. For the rest of the cases, either a chief resident (PGY4 or PGY5) or a Hepatobiliary Surgery fellow was the assistant. Totally laparoscopic cases typically used 6 trocars (four $12 \mathrm{~mm}$ trocars and two $5 \mathrm{~mm}$ trocars) while robotic-assisted typically used 5 trocars (four robotic $8 \mathrm{~mm}$ trocars and one $12 \mathrm{~mm}$ assistant trocars). The decision to use robotic assistance largely depended on institutional robot availability. Initially, the Department had access to the robot 2 days per month, which limited the amount of robotic cases that could be scheduled. There were no specific selection criteria for patients to be considered eligible for MIPD. All patients underwent pancreaticojejunostomy in a 2-layered duct-to-mucosa fashion. MIPDs that were converted to open were still considered MIPD for the analysis in an intentionto-treat manner.

Patients able to understand and willing to sign an IRBapproved informed consent document (IRB \#201908115) who underwent MIPD were compared to patients who underwent OPD during the same time frame (i.e., from April 2016 to February 2020) in an intention-to-treat fashion. Multivariable logistic regression was used to examine the association of covariates age, sex, race, comorbidities (coronary artery disease [CAD], Chronic Obstructive Pulmonary disease [COPD], diabetes [DM], obesity) American Society of Anesthesiologists (ASA) class, history of pancreatitis, pancreatic duct diameter, pancreatic adenocarcinoma, vascular resection, neoadjuvant therapy status (preoperative chemotherapy or radiation therapy), and tumor stage with ideal outcome; 'MIPD $\times$ pancreatic duct diameter' was used as an interaction term within the model to test for effect modification. MIPD patients with dilated pancreatic ducts were 1:3 propensity score matched to OPD patients with dilated ducts and outcomes compared. Likewise, MIPD patients with nondilated pancreatic ducts were $1: 3$ propensity score matched to OPD patients with non-dilated ducts and outcomes compared. Patients were matched by age, sex, race, comorbidities (coronary artery disease [CAD], Chronic Obstructive Pulmonary disease [COPD], diabetes [DM], obesity) ASA class, history of pancreatitis, pancreatic adenocarcinoma, vascular resection, neoadjuvant therapy status (preoperative chemotherapy or radiation therapy), and tumor stage using a nearest neighbor matching algorithm. Unpaired Student's $t$-tests were used to compare continuous variables and outcomes, whereas chi-square tests were used to compare categorical variables and outcomes. All p-values were 2-sided, and a $\mathrm{p}$ value less than 0.05 was considered statistically significant. All statistical analysis was performed using SAS version 9.4 (Cary, NC).

\section{Results}

\section{Total cohort}

In total, 371 patients underwent PD from April 2016 to February 2020. Seventy-four patients (19.9\%) underwent MIPD, and 297 patients (80.1\%) underwent OPD (Table 1). Twenty-two (29.7\%) of the MIPDs were robotic-assisted.
Table 1 Characteristics of patients undergoing minimally invasive or open pancreatoduodenectomy $(n=371)$

\begin{tabular}{lccc}
\hline Characteristic & $\mathrm{N}($ column $\%)$ or mean $(\mathrm{SD})$ & & $p$ value \\
\cline { 2 - 4 } & Open $(n=297,80.1 \%)$ & $\mathrm{MIS}(n=74,19.9 \%)$ & \\
\hline Demographics & & & \\
Age $>65$ years & $165(55.6 \%)$ & $39(52.7 \%)$ & 0.659 \\
Male & $159(53.5 \%)$ & $32(43.2 \%)$ & 0.113 \\
White & $257(86.5 \%)$ & $65(87.8 \%)$ & 0.767 \\
Comorbidities & & & 0.388 \\
Obese & $83(27.9 \%)$ & $17(22.9 \%)$ & 0.755 \\
Coronary artery disease & $36(12.1 \%)$ & $8(10.8 \%)$ & 0.136 \\
Diabetes & $99(33.3 \%)$ & $18(24.3 \%)$ & 0.344 \\
COPD & $26(8.8 \%)$ & $4(5.4 \%)$ & 0.832 \\
History of pancreatitis & $43(14.5 \%)$ & $10(13.5 \%)$ & 0.940 \\
ASA 3 & $170(57.2 \%)$ & $42(56.8 \%)$ & \\
Operative details & $182(61.3 \%)$ & & 0.617 \\
Pancreatic adenocarcinoma & $4.4(2.2)$ & $43(58.1 \%)$ & 0.068 \\
Pancreatic duct size, mm & $178(59.9 \%)$ & $3.8(2.2)$ & 0.078 \\
Neoadjuvant therapy & $81(27.3 \%)$ & $36(48.7 \%)$ & $\mathbf{0 . 0 4 9}$ \\
Vascular resection & $171(57.6 \%)$ & $12(16.2 \%)$ & $\mathbf{0 . 0 4 4}$ \\
T3 or T4 tumor & $33(44.6 \%)$ & \\
\hline
\end{tabular}

$[$ Bold $]=$ Statistically significant $p$ value $(p<0.05)$ 
Seventeen patients $(22.9 \%)$ experienced unplanned conversion to open. Reasons for conversion included prohibitive adhesions/scarring $(n=10)$ and failure to make progress due to difficulty identifying key structures $(n=7)$. Patients who were converted from MIPD to an open procedure were less likely to have T3/T4 tumors (11.8 vs $54.4 \%, p=0.002$ ) and more likely to have been started laparoscopically versus robot-assisted (30.77 vs $4.55 \%, p=0.014$ ). There were no statistically significant differences in duct size or need for vascular resection between converted and non-converted patients. (See Supplemental Table A). Three MIPD patients died within 90 days of surgery and 17 patients in the OPD group died within 90 days postoperatively (4.1 vs $5.7 \%$, $p=0.359)$.

Forty-five $(60.8 \%)$ of the MIPD patients and 197 $(66.3 \%)$ of the OPD patients had dilated $(\geq 3 \mathrm{~mm})$ pancreatic ducts $(p=0.372)$. Patients who underwent MIPD were significantly less likely to have T3/T4 tumors (44.6 vs $57.6 \%, p=0.044)$ and less likely to undergo venous vascular resection (16.2 vs $27.3 \%, p=0.049$ ) (Table 1 ) compared to OPD patients. Patients who underwent MIPD had significantly longer operative times (470.2 vs $374.3 \mathrm{~min}$, $p<0.001)$ as well as significantly less intraoperative blood loss (298.6 vs $429.8 \mathrm{ml}, p=0.016$ ) (Table 2).
Table 2 Perioperative and pathologic outcomes of patients undergoing minimally invasive or open pancreaticoduodenectomy $(n=371)$

\begin{tabular}{|c|c|c|c|}
\hline \multirow[t]{2}{*}{ Outcomes } & \multicolumn{2}{|c|}{ No. (column \%) or mean (SD) } & \multirow[t]{2}{*}{$p$ value } \\
\hline & Open $(n=297,80.05 \%)$ & MIS $(n=74,19.95 \%)$ & \\
\hline \multicolumn{4}{|l|}{ Postoperative complications } \\
\hline Delayed gastric emptying & $65(21.9 \%)$ & $18(24.3 \%)$ & 0.652 \\
\hline Postoperative bleeding/anemia & $98(33.0 \%)$ & $21(28.4 \%)$ & 0.446 \\
\hline Surgical site infection & $20(6.7 \%)$ & $5(6.8 \%)$ & 0.994 \\
\hline Any pancreatic fistula & $50(16.8 \%)$ & $16(21.6 \%)$ & 0.335 \\
\hline Grade $\mathrm{B} / \mathrm{C}$ pancreatic fistula & $38(12.8 \%)$ & $8(10.8 \%)$ & 0.643 \\
\hline Pancreatic fistula $\geq$ MAGS grade 3 & $25(8.4 \%)$ & $7(9.5 \%)$ & 0.775 \\
\hline Organ space infection & $29(9.8 \%)$ & $9(12.2 \%)$ & 0.543 \\
\hline Arrhythmia & $14(4.7 \%)$ & $2(2.7 \%)$ & 0.446 \\
\hline Myocardial infarction & $3(1.0 \%)$ & $0(0 \%)$ & 0.385 \\
\hline Failure to thrive & $13(4.4 \%)$ & $1(1.4 \%)$ & 0.222 \\
\hline Bacteremia & $12(4.0 \%)$ & $2(2.7 \%)$ & 0.589 \\
\hline DVT-PE & $16(5.4 \%)$ & $3(4.1 \%)$ & 0.642 \\
\hline Pneumonia & $10(3.4 \%)$ & $2(2.7 \%)$ & 0.773 \\
\hline $\mathrm{C}$ diff colitis & $2(0.7 \%)$ & $1(1.4 \%)$ & 0.560 \\
\hline Urinary retention & $60(20.2 \%)$ & $8(10.8 \%)$ & 0.062 \\
\hline Urinary tract infection & $10(3.4 \%)$ & $2(2.7 \%)$ & 0.773 \\
\hline Any complication & $216(72.7 \%)$ & $47(63.5 \%)$ & 0.119 \\
\hline Non-severe complication ( $<$ MAGS grade 3$)$ & $119(40.1 \%)$ & $22(29.7 \%)$ & 0.101 \\
\hline Severe complication ( $\geq$ MAGS grade 3 ) & $97(32.7 \%)$ & $25(33.8 \%)$ & 0.854 \\
\hline Mean postoperative length of stay, days & $10.9(11.5)$ & $10.6(13.4)$ & 0.834 \\
\hline Composite length of stay [24], days & $12.6(12.7)$ & $10.9(13.4)$ & 0.318 \\
\hline 90-Day readmission & $90(30.30 \%)$ & $15(20.3 \%)$ & 0.087 \\
\hline 90-Day mortality & $17(5.72 \%)$ & $3(4.1 \%)$ & 0.569 \\
\hline Ideal outcome & $47(15.8 \%)$ & $15(20.3 \%)$ & 0.359 \\
\hline \multicolumn{4}{|l|}{ Operative details } \\
\hline Surgery time, minutes & $374.3(130.3)$ & $470.2(119.8)$ & $<0.001$ \\
\hline Intraoperative blood loss, $\mathrm{ml}$ & $429.8(445.3)$ & $298.6(262.6)$ & 0.001 \\
\hline Intraoperative transfusions & $46(15.5 \%)$ & $7(9.5 \%)$ & 0.185 \\
\hline \multicolumn{4}{|l|}{ Pathology results } \\
\hline Lymph nodes removed & $22.4(9.5)$ & $20.5(6.5)$ & 0.061 \\
\hline Lymph nodes positive & $3.7(5.3)$ & $3.1(4.6)$ & 0.447 \\
\hline Positive margins* & $81(27.3 \%)$ & $21(28.4 \%)$ & 0.849 \\
\hline
\end{tabular}

$[$ Bold $]=$ Statistically significant $p$ value $(p<0.05)$

MAGS modified accordion grading system

* Margin $<1 \mathrm{~mm}$ considered positive 
The rates of any pancreatic fistula (21.6 vs $16.8 \%$, $p=0.335)$, clinically relevant pancreatic fistula (10.8 vs $12.8 \%, 0.643)$, or pancreatic fistula resulting in a severe complication (9.5 vs $8.4 \%, p=0.775$ ) were not significantly different between MIPD and OPD. There were also no differences in pathologic outcomes, with similar numbers of lymph nodes retrieved ( 20.5 vs $22.4, p=0.061)$ and similar rates of positive margins ( 28.4 vs $27.3 \%, p=0.849$ ) in MIPD and OPD patients.

In total, 15 (20.3\%) patients had Ideal Outcome with MIPD and $47(15.8 \%)$ patients had ideal outcome with OPD, $(p=0.359)$.

In a multivariate analysis, the combination of operative approach (MIPD vs OPD) and pancreatic duct size (non-dilated $[<3 \mathrm{~mm}]$ vs dilated $[\geq 3 \mathrm{~mm}]$ ) had a significant interaction with respect to these variables' association with ideal outcome $(p=0.017)$. After subcategorizing

Table 3 Multivariate analysis of factors associated with ideal outcome after pancreaticoduodenectomy

\begin{tabular}{|c|c|c|c|}
\hline Variables & Odds ratio & $95 \% \mathrm{CI}$ & $p$ value \\
\hline \multicolumn{4}{|l|}{ Age } \\
\hline Age $\leq 65$ years & [Reference] & & \\
\hline Age $>65$ years & 1.30 & $0.71-2.37$ & 0.393 \\
\hline \multicolumn{4}{|l|}{ Sex } \\
\hline Female & [Reference] & & \\
\hline Male & 0.99 & $0.56-1.76$ & 0.977 \\
\hline \multicolumn{4}{|l|}{ Race } \\
\hline White & [Reference] & & \\
\hline Non-white & 0.38 & $0.13-1.14$ & 0.084 \\
\hline \multicolumn{4}{|l|}{ Comorbidities } \\
\hline Coronary artery disease & 0.47 & $0.15-1.47$ & 0.1944 \\
\hline Diabetes & 0.97 & $0.51-1.83$ & 0.912 \\
\hline COPD & 1.46 & $0.50-4.22$ & 0.487 \\
\hline Obesity & 0.73 & $0.37-1.47$ & 0.384 \\
\hline History of pancreatitis & 1.53 & $0.71-3.32$ & 0.282 \\
\hline ASA class $\geq 3$ & 0.64 & $0.35-1.17$ & 0.145 \\
\hline \multicolumn{4}{|l|}{ Operative factors } \\
\hline Pancreatic adenocarcinoma & 0.79 & $0.41-1.52$ & 0.480 \\
\hline Neoadjuvant therapy & 1.53 & $0.80-2.93$ & 0.198 \\
\hline Vascular resection & 0.69 & $0.32-1.46$ & 0.325 \\
\hline $\mathrm{T} 3$ or T4 tumor & 0.63 & $0.35-1.12$ & 0.114 \\
\hline \multicolumn{4}{|l|}{ Approach and duct size } \\
\hline Open with $<3 \mathrm{~mm}$ duct & [Reference] & & \\
\hline Open with $\geq 3 \mathrm{~mm}$ duct & 1.44 & $0.74-2.80$ & 0.282 \\
\hline MIS with $<3 \mathrm{~mm}$ duct & 0.41 & $0.09-1.89$ & 0.254 \\
\hline MIS with $\geq 3 \mathrm{~mm}$ duct & 2.38 & $1.08-5.26$ & $\mathbf{0 . 0 3 3}$ \\
\hline
\end{tabular}

[Bold $]=$ Statistically significant $p$ value $(p<0.05)$

Ideal outcome defined as patients having no complications (including no death and no readmission), a length of hospital stay equal to or less than the overall median length of stay (7 days), and negative (R0) margins operative approach into MIPD with or without dilated pancreatic ducts and OPD with or without dilated pancreatic ducts, MIPD in patients with dilated pancreatic ducts was independently associated with increased ideal outcome (OR $=2.38, p=0.033$ ) (Table 3$)$. However, MIPD in patients with non-dilated pancreatic ducts was not associated with ideal outcome $(\mathrm{OR}=0.41, p=0.254)$.

\section{Propensity score matched patients}

Given the effect modification of dilated pancreatic ducts on the association of MIPD with ideal outcome, patients who underwent pancreaticoduodenectomy were divided into those with dilated pancreatic ducts $(\geq 3 \mathrm{~mm})$ and those with non-dilated pancreatic ducts $(<3 \mathrm{~mm})$ in order to propensity score match MIPD patients to OPD patients within the same duct diameter category. The 45 patients with dilated pancreatic ducts who underwent MIPD were propensity score matched (1:3) to 135 patients with dilated pancreatic ducts who underwent OPD during the study time period (Table 4). MIPD patients with dilated pancreatic ducts had significantly longer operative times ( 445.9 vs $344.9 \mathrm{~min}, p<0.001$ ), lower overall complication rate $(51.1 \mathrm{vs} 75.6 \%, p=0.002)$ and lower readmission rate (11.1 vs $30.4 \%, p=0.010$ ) compared to matched OPD patients. There were no statistically significant differences in intraoperative blood loss ( 258.9 vs $323.1 \mathrm{ml}, p=0.099$ ), postoperative bleeding/anemia complications ( 24.4 vs $31.1 \%, p=0.396$ ), postoperative length of stay ( 9.1 vs 10.3 days, $p=0.502$ ), rates of any pancreatic fistula (13.3 vs $17.8 \%, p=0.488)$, rates of clinically relevant pancreatic fistula (6.7 vs $15.6 \%, p=0.129)$ or rates of pancreatic fistula associated with severe complications (6.7 vs $11.1 \%, p=0.389)$. The incidence of positive margins (28.9 vs $23.7 \%, p=0.487)$ and average number of lymph nodes retrieved ( 21.6 vs $23.3, p=0.322$ ) were similar between matched MIPD and OPD patients with dilated pancreatic ducts. Patients with dilated ducts who underwent MIPD had significantly higher rates of ideal outcome than patients who underwent OPD (28.9 vs $14.8 \%, p=0.0356$ ) (Table 5).

The 29 patients with non-dilated pancreatic ducts who underwent MIPD were propensity score matched (1:3) to 87 patients with non-dilated pancreatic ducts who underwent OPD (Table 6).

MIPD patients with non-dilated pancreatic ducts had significantly longer operative times (493.4 vs $398.8 \mathrm{~min}$, $p=0.001)$ but there were no other significant differences in perioperative outcomes compared to matched OPD patients (Table 7). Likewise, the incidence of positive margins (27.6 vs $22.9 \%, p=0.616)$ and the number of lymph nodes removed (18.8 vs $21.2, p=0.121)$ were not significantly different between MIPD and OPD patients with non-dilated pancreatic ducts. In patients with small ducts, there was no significant difference in ideal outcome after MIPD vs OPD (6.9 vs $19.5 \%, p=0.111)$. 
Table 4 Characteristics of 3:1 propensity score-matched patients with pancreatic duct diameter $\geq 3 \mathrm{~mm}$ undergoing open versus minimally invasive pancreaticoduodenectomy $(n=180)$

\begin{tabular}{lccc}
\hline Characteristic & \multicolumn{2}{l}{ No. (column \%) or mean (SD) } & $p$ value \\
\cline { 2 - 4 } & Open $(n=135,75 \%)$ & MIS $(n=45,25 \%)$ & \\
\hline Demographics & & & \\
Age $>65$ years & $65(59.3 \%)$ & $23(51.1 \%)$ & 0.339 \\
Male & $122(90.4 \%)$ & $19(42.2 \%)$ & 0.490 \\
White & & $39(86.7 \%)$ & 0.484 \\
Comorbidities & $30(22.2 \%)$ & & 0.754 \\
Obese & $13(9.6 \%)$ & $9(20.0 \%)$ & 0.883 \\
Coronary artery disease & $32(23.7 \%)$ & $4(8.9 \%)$ & 0.608 \\
Diabetes & $3(2.2 \%)$ & $9(20.0 \%)$ & 1.000 \\
COPD & $19(14.1 \%)$ & $1(2.2 \%)$ & 0.901 \\
History of pancreatitis & $70(51.9 \%)$ & $6(13.3 \%)$ & 0.931 \\
ASA class $\geq 3$ & $80(59.3 \%)$ & $23(51.1 \%)$ & \\
Operative details & $66(48.9 \%)$ & $26(57.8 \%)$ & 0.861 \\
Pancreatic adenocarcinoma & $21(15.6 \%)$ & $22(48.9 \%)$ & 1.000 \\
Neoadjuvant therapy & $62(45.9 \%)$ & $7(15.6 \%)$ & 1.000 \\
Vascular resection & & $20(44.4 \%)$ & 0.863 \\
T3 or T4 tumor & &
\end{tabular}

\section{Discussion}

In our study, MIPD appears to be safe overall with similar outcomes compared to OPD. We sought to understand which patients (if any) benefit from MIPD in order to allow surgeons to better optimize preoperative patient selection. Our study showed that the minimally invasive approach benefited patients with dilated pancreatic ducts at our institution. In our experience, MIPD was associated with significantly fewer overall complications, lower readmission rate and higher likelihood of an Ideal Outcome compared to OPD in patients with dilated pancreatic ducts. We did not observe a significant difference in overall complication rate or readmissions in patients with non-dilated ducts; however, MIPD appears safe in this patient subset as well, with similar rates of complications and severe complications, and no significant difference in 90-day mortality.

Pancreatic fistula is the "Achilles Heel" of pancreaticoduodenectomy. Fortunately, most patients do not experience pancreatic fistula after pancreaticoduodenectomy, and perhaps these are the patients that benefit most from the minimally invasive approach. Most studies have not found a difference in the rate of pancreatic fistula with MIPD compared to OPD [3-7. Although there is some recent literature to suggest a reduction in clinically relevant pancreatic fistula with robotic pancreaticoduodenectomy compared to OPD, it is unlikely that MIPD will "solve" the problem of pancreatic fistula [19]. However, for the majority of patients undergoing pancreaticoduodenectomy who are known to be at lower risk of pancreatic fistula preoperatively (i.e., have dilated pancreatic ducts), MIPD may perhaps improve recovery.
There have been three randomized trials comparing MIPD to OPD. In a randomized study comparing 32 patients undergoing laparoscopic $\mathrm{PD}$ to 32 patients undergoing OPD, Palanivelu et al. demonstrated significantly reduced postoperative length of stay in patients undergoing laparoscopic PD as well as significantly reduced intraoperative blood loss, less perioperative blood transfusions, and increased operative time compared to OPD patients [5]. In a study by Poves et al. which randomized 32 patients to laparoscopic pancreaticoduodenectomy and 29 patients to OPD, laparoscopic PD patients had significantly fewer severe complications as well as significantly fewer patients requiring prolonged postoperative length of stay compared to OPD, but also had significantly increased operative times [6]. In a multicenter randomized controlled trial from the Netherlands, van Hilst et al. found no significant differences in postoperative outcomes, but the trial was stopped prematurely due to a nonstatistically significant trend toward increased mortality in the laparoscopic group [7]. Meta-analyses of the these small randomized trials suggest that MIPD is associated with significantly increased operative times with significantly decreased intraoperative blood loss without an observed difference in other postoperative outcomes [20, 21]. None of these randomized trials excluded patients at high risk of pancreatic fistula, a complication which could perhaps limit the potential benefit of MIPD on recovery. Likewise, these randomized trials have not addressed the issue of duct size driving postoperative outcomes. Our rate of clinically relevant pancreatic fistula in MIPD patients was over 5 times higher in patients with non-dilated ducts compared to patients with dilated ducts. We suspect that this higher rate of clinically relevant pancreatic fistula could mitigate 
Table 5 Perioperative outcomes of 3:1 propensity score-matched patients with pancreatic duct diameter $\geq 3 \mathrm{~mm}$ undergoing open versus minimally invasive pancreaticoduodenectomy $(n=180)$

\begin{tabular}{|c|c|c|c|}
\hline & \multicolumn{2}{|c|}{ No. (column \%) or mean (SD) } & \multirow[b]{2}{*}{$p$ value } \\
\hline & Open $(n=135,75 \%)$ & $\operatorname{MIS}(n=45,25 \%)$ & \\
\hline \multicolumn{4}{|l|}{ Postoperative complications } \\
\hline Delayed gastric emptying & $31(22.9 \%)$ & $10(22.2 \%)$ & 0.918 \\
\hline Postoperative bleeding/anemia & $42(31.1 \%)$ & $11(24.4 \%)$ & 0.396 \\
\hline Surgical site infection & $10(7.4 \%)$ & $1(2.2 \%)$ & 0.209 \\
\hline Urinary retention & $29(21.5 \%)$ & $4(8.9 \%)$ & 0.059 \\
\hline Any pancreatic fistula & $24(17.8 \%)$ & $6(13.3 \%)$ & 0.488 \\
\hline Grade B/C pancreatic fistula & $21(15.6 \%)$ & $3(6.7 \%)$ & 0.129 \\
\hline Pancreatic fistula $\geq$ MAGS grade 3 & $15(11.1 \%)$ & $3(6.7 \%)$ & 0.389 \\
\hline Organ space infection & $13(9.6 \%)$ & $4(8.9 \%)$ & 0.883 \\
\hline Arrhythmia & $7(5.2 \%)$ & $2(4.4 \%)$ & 0.844 \\
\hline Myocardial infarction & $2(1.5 \%)$ & $0(0.0 \%)$ & 0.412 \\
\hline Failure to thrive & $6(4.4 \%)$ & $0(0.0 \%)$ & 0.150 \\
\hline Bacteremia & $7(5.2 \%)$ & $2(4.4 \%)$ & 0.844 \\
\hline $\mathrm{C}$ diff colitis & $2(1.5 \%)$ & $1(2.2 \%)$ & 0.737 \\
\hline DVT-PE & $9(6.7 \%)$ & $2(4.4 \%)$ & 0.589 \\
\hline Pneumonia & $4(2.9 \%)$ & $1(2.2 \%)$ & 0.793 \\
\hline Urinary tract infection & $6(4.4 \%)$ & $0(0.0 \%)$ & 0.150 \\
\hline Any complication & $102(75.6 \%)$ & $23(51.1 \%)$ & 0.002 \\
\hline Non-severe complication (<MAGS grade 3 ) & $55(40.7 \%)$ & $12(26.7 \%)$ & 0.091 \\
\hline Severe complication ( $\geq$ MAGS grade 3 ) & $47(34.8 \%)$ & $11(24.4 \%)$ & 0.197 \\
\hline Postoperative length of stay, days & $10.3(7.4)$ & $9.1(10.7)$ & 0.502 \\
\hline Composite length of stay [24], days & $12.3(9.2)$ & $9.4(10.7)$ & 0.074 \\
\hline 90-Day readmission & $41(30.4 \%)$ & $5(11.1 \%)$ & $\mathbf{0 . 0 1 0}$ \\
\hline 90-Day mortality & $7(5.2 \%)$ & $2(4.4 \%)$ & 0.844 \\
\hline Ideal outcome & $20(14.8 \%)$ & $13(28.9 \%)$ & $\mathbf{0 . 0 3 5}$ \\
\hline \multicolumn{4}{|l|}{ Operative details } \\
\hline Surgery time, minutes & $344.9(120.6)$ & $445.9(124.7)$ & $<0.001$ \\
\hline Intraoperative blood loss, $\mathrm{ml}$ & $323.1(280.4)$ & $258.9(199.5)$ & 0.186 \\
\hline Intraoperative transfusions & $15(11.1 \%)$ & $3(6.7 \%)$ & 0.389 \\
\hline \multicolumn{4}{|l|}{ Pathology results } \\
\hline Lymph nodes removed & $23.3(9.3)$ & $21.6(7.3)$ & 0.322 \\
\hline Lymph nodes positive & $3.9(4.9)$ & $3.5(5.3)$ & 0.664 \\
\hline Positive margins* & $32(23.7 \%)$ & $13(28.9 \%)$ & 0.487 \\
\hline
\end{tabular}

[Bold $]=$ Statistically significant $p$ value $(p<0.05)$

MAGS modified accordion grading system

*Margin $<1 \mathrm{~mm}$ considered positive the benefit that MIPD offers in terms of 90-day readmission rate and overall rate of ideal outcome.

Our findings in the overall patient cohort are similar to those reported in the literature with regards to increased operative times and decreased intraoperative blood loss [3-5]. Nassour et al. demonstrated that MIPD was associated with increased 30-day readmission using the NSQIP pancreatectomy database [22]. However, we observed a statistically significant reduction in 90-day readmission in the MIPD group of patients with dilated pancreatic ducts. The overall complication rate in our study was around $70 \%$, which is somewhat higher than other published studies in the literature. However, the majority of these complications were MAGS non-severe. We have previously shown that the incidence of MAGS non-severe complications vary widely depending on the definition and method of complication gathering, which makes it challenging to compare the rates of lesser severe complications between studies at different institutions [15]. Most complications in the current study, such as the rates of pancreatic fistulas (17.8\%), delayed gastric emptying (22.4\%), and postoperative bleeding/anemia complications (32.1\%), were similar to previously published results. 
Table 6 Characteristics of $3: 1$ propensity score-matched patients with pancreatic duct diameter $<3 \mathrm{~mm}$ undergoing open versus minimally invasive pancreaticoduodenectomy $(n=116)$

\begin{tabular}{llcl}
\hline Characteristic & \multicolumn{2}{l}{ No. (column \%) or mean (SD) } & $p$ value \\
\cline { 2 - 4 } & Open $(n=87,75 \%)$ & MIS $(n=29,25 \%)$ & \\
\hline Demographics & & & \\
Age $>65$ years & $46(52.9 \%)$ & $16(55.2 \%)$ & 0.829 \\
Male & $41(47.1 \%)$ & $13(44.8 \%)$ & 0.829 \\
White & $75(86.2 \%)$ & $26(89.7 \%)$ & 0.632 \\
Comorbidities & & & 0.905 \\
Obese & $25(28.7 \%)$ & $8(27.6 \%)$ & 0.742 \\
Coronary artery disease & $10(11.5 \%)$ & $4(13.8 \%)$ & 0.734 \\
Diabetes & $30(34.5 \%)$ & $9(31.0 \%)$ & 0.703 \\
COPD & $7(8.1 \%)$ & $3(10.3 \%)$ & 0.873 \\
History of pancreatitis & $11(12.6 \%)$ & $4(13.8 \%)$ & 0.384 \\
ASA class $\geq 3$ & $49(56.3 \%)$ & $19(65.5 \%)$ & 1.000 \\
Operative details & & & 0.451 \\
Pancreatic adenocarcinoma & $51(58.6 \%)$ & $17(58.6 \%)$ & 0.441 \\
Neoadjuvant therapy & $49(56.3 \%)$ & $14(48.3 \%)$ & 0.391 \\
Vascular resection & $21(24.1 \%)$ & $5(17.2 \%)$ & \\
T3 or T4 tumor & $47(54.0 \%)$ & & \\
\hline
\end{tabular}

Our study had several limitations. While our study was prospective, it was not randomized and is thus subject to selection bias. We report here our experience with our first 74 MIPD at our institution, and thus our sample size is relatively small. However, these results demonstrate that MIPD can be instituted safely with acceptable outcomes at a high-volume pancreatectomy center. A limitation in comparing intraoperative blood loss between MIPD and OPD may reside in the differences inherent to the measurement of intraoperative blood loss in patients during open versus minimally invasive/laparoscopic surgery. Propensity score matching attempts to equally distribute variables in matched patients, but it is possible that unmeasured variables could confound the analysis. Despite these limitations, our methodology using propensity score matching of prospectively gathered observational data is likely to be highly predictive of the results from independently performed randomized, controlled clinical trials [23].

In conclusion, MIPD is safe with comparable perioperative and pathologic outcomes to OPD. In our early experience, patients with dilated pancreatic ducts (at low risk for pancreatic fistula) appear to derive the most benefit in terms of reduced complications, lower readmission rates and higher likelihood of ideal outcome. Due to its complexity and steep learning curve, these results may help guide preoperative patient selection and indications during the adoption of MIPD. Further prospective study is needed to confirm the validity and generalizability of these findings.

\section{Disclosures}

The following authors have no conflicts of interest or financial ties to disclose: Dr. Heidy Cos, Dr. Michael T. LeCompte, Dr. Sanket Srinivasa, Dr. Jorge Zarate Rodriguez, Ms. Cheryl A. Woolsey, Mr. Siddarth Patel, Dr. Adeel Khan, Dr. Ryan C. Fields, Dr. Maria B. Doyle, Dr. William C. Chapman, Dr. Steven M. Strasberg, Dr. William G. Hawkins, Dr. Chet W. Hammill and Dr. Dominic E. Sanford. 
Table 7 Perioperative outcomes of 3:1 propensity score-matched patients with pancreatic duct diameter $<3 \mathrm{~mm}$ undergoing open versus minimally invasive pancreaticoduodenectomy $(n=116)$

\begin{tabular}{|c|c|c|c|}
\hline \multirow[t]{2}{*}{ Complications } & \multicolumn{2}{|c|}{ No. (column \%) or mean (SD) } & \multirow[t]{2}{*}{$p$ value } \\
\hline & Open $(n=87,75 \%)$ & $\operatorname{MIS}(n=29,25 \%)$ & \\
\hline \multicolumn{4}{|l|}{ Postoperative complications } \\
\hline DGE & $18(20.7 \%)$ & $8(27.6 \%)$ & 0.441 \\
\hline Postoperative bleeding/anemia & $31(35.6 \%)$ & $10(34.5 \%)$ & 0.911 \\
\hline Surgical site infection & $4(4.6 \%)$ & $4(13.8 \%)$ & 0.091 \\
\hline Urinary retention & $18(20.7 \%)$ & $4(13.8 \%)$ & 0.412 \\
\hline Any pancreatic fistula & $16(18.4 \%)$ & $10(34.5 \%)$ & 0.072 \\
\hline Grade $\mathrm{B} / \mathrm{C}$ pancreatic fistula & $10(11.5 \%)$ & $5(17.2 \%)$ & 0.424 \\
\hline Pancreatic fistula $\geq$ grade 3 & $7(8.1 \%)$ & $4(13.8 \%)$ & 0.360 \\
\hline Organ space infection & $11(12.6 \%)$ & $5(17.2 \%)$ & 0.534 \\
\hline Arrhythmia & $5(5.8 \%)$ & $0(0.0 \%)$ & 0.187 \\
\hline Myocardial infarction & $1(1.2 \%)$ & $0(0.0 \%)$ & 0.562 \\
\hline Failure to thrive & $4(4.6 \%)$ & $1(3.5 \%)$ & 0.792 \\
\hline Bacteremia & $3(3.5 \%)$ & $0(0.0 \%)$ & 0.311 \\
\hline DVT-PE & $4(4.6 \%)$ & $1(3.5 \%)$ & 0.792 \\
\hline Pneumonia & $3(3.5 \%)$ & $1(3.5 \%)$ & 1.000 \\
\hline Urinary tract infection & $4(4.6 \%)$ & $2(6.9 \%)$ & 0.628 \\
\hline Any complication & $63(72.4 \%)$ & $24(82.8 \%)$ & 0.265 \\
\hline Non-severe complication (<MAGS grade 3 ) & $33(37.9 \%)$ & $10(34.5 \%)$ & 0.739 \\
\hline Severe complication ( $\geq$ MAGS grade 3 ) & $30(34.5 \%)$ & $14(48.3 \%)$ & 0.185 \\
\hline Postoperative length of stay, days & $12.7(17.9)$ & $12.9(16.9)$ & 0.943 \\
\hline Composite length of stay [24], days & $13.9(17.9)$ & $13.5(16.8)$ & 0.894 \\
\hline 90-Day readmission & $30(34.5 \%)$ & $10(34.5 \%)$ & 1.000 \\
\hline 90-Day mortality & $8(9.2 \%)$ & $1(3.5 \%)$ & 0.316 \\
\hline Ideal outcome & $17(19.5 \%)$ & $2(6.9 \%)$ & 0.111 \\
\hline \multicolumn{4}{|l|}{ Operative details } \\
\hline Surgery time, minutes & $398.8(132.1)$ & $493.4(109.7)$ & $<0.001$ \\
\hline Intraoperative blood loss, $\mathrm{ml}$ & $522.7(578.1)$ & $362.5(334.9)$ & 0.076 \\
\hline Intraoperative transfusions & $18(20.7 \%)$ & $4(13.8 \%)$ & 0.412 \\
\hline \multicolumn{4}{|l|}{ Pathology results } \\
\hline Lymph nodes removed & $21.2(9.4)$ & $18.8(4.8)$ & 0.121 \\
\hline Lymph nodes positive & $3.4(5.9)$ & $2.5(3.4)$ & 0.363 \\
\hline Positive margins* & $20(22.9 \%)$ & $8(27.6 \%)$ & 0.616 \\
\hline
\end{tabular}

MAGS modified accordion grading system

* Margin $<1 \mathrm{~mm}$ considered positive
Supplementary Information The online version contains supplementary material available at https://doi.org/10.1007/s00464-021-08611-x.

Acknowledgements Mr. Gregory Williams is supported by the SPORE Grant 5P50 CA196510.

\section{References}

1. Boone BA, Zenati M, Hogg ME et al (2015) Assessment of quality outcomes for robotic pancreaticoduodenectomy: identification of the learning curve. JAMA Surg 150(5):416-422

2. Nagakawa $Y$, Nakamura $Y$, Honda $G$ et al (2018) Learning curve and surgical factors influencing the surgical outcomes during the initial experience with laparoscopic pancreaticoduodenectomy. J Hepatobiliary Pancreat Sci 25(11):498-507

3. Asbun HJ, Stauffer JA (2012) Laparoscopic vs open pancreaticoduodenectomy: overall outcomes and severity of complications using the Accordion Severity Grading System. J Am Coll Surg 215(6):810-819

4. Croome KP, Farnell MB, Que FG et al (2014) Total laparoscopic pancreaticoduodenectomy for pancreatic ductal adenocarcinoma: oncologic advantages over open approaches? Ann Surg 260(4):633-638

5. Palanivelu C, Senthilnathan P, Sabnis SC et al (2017) Randomized clinical trial of laparoscopic versus open pancreatoduodenectomy for periampullary tumours. Br J Surg 104(11):1443-1450

6. Poves I, Burdio F, Morato O et al (2018) Comparison of perioperative outcomes between laparoscopic and open approach for 
pancreatoduodenectomy: the PADULAP randomized controlled trial. Ann Surg 268(5):731-739

7. van Hilst J, de Rooij T, Bosscha K et al (2019) Laparoscopic versus open pancreatoduodenectomy for pancreatic or periampullary tumours (LEOPARD-2): a multicentre, patient-blinded, randomised controlled phase 2/3 trial. Lancet Gastroenterol Hepatol 4(3):199-207

8. Kutlu OC, Lee JE, Katz MH et al (2018) Open pancreaticoduodenectomy case volume predicts outcome of laparoscopic approach: a population-based analysis. Ann Surg 267(3):552-560

9. McMillan MT, Christein JD, Callery MP et al (2016) Comparing the burden of pancreatic fistulas after pancreatoduodenectomy and distal pancreatectomy. Surgery 159(4):1013-1022

10. McMillan MT, Vollmer CM Jr, Asbun HJ et al (2016) The characterization and prediction of ISGPF grade $\mathrm{C}$ fistulas following pancreatoduodenectomy. J Gastrointest Surg 20(2):262-276

11. Callery MP, Pratt WB, Kent TS, Chaikof EL, Vollmer CM Jr (2013) A prospectively validated clinical risk score accurately predicts pancreatic fistula after pancreatoduodenectomy. J Am Coll Surg 216(1):1-14

12. Kantor O, Talamonti MS, Pitt HA et al (2017) Using the NSQIP pancreatic demonstration project to derive a modified fistula risk score for preoperative risk stratification in patients undergoing pancreaticoduodenectomy. J Am Coll Surg 224(5):816-825

13. Martin AN, Narayanan S, Turrentine FE, Bauer TW, Adams RB, Zaydfudim VM (2018) Pancreatic duct size and gland texture are associated with pancreatic fistula after pancreaticoduodenectomy but not after distal pancreatectomy. PLoS ONE 13(9):e0203841

14. Mungroop TH, van Rijssen LB, van Klaveren D et al (2017) Alternative fistula risk score for pancreatoduodenectomy (a-FRS): design and international external validation. Ann Surg 269(5):937-943

15. Sanford DE, Woolsey CA, Hall BL et al (2014) Variations in definition and method of retrieval of complications influence outcomes statistics after pancreatoduodenectomy: comparison of NSQIP with non-NSQIP methods. J Am Coll Surg 219(3):407-415
16. Strasberg SM, Linehan DC, Hawkins WG (2009) The accordion severity grading system of surgical complications. Ann Surg 250(2):177-186

17. Clavien PA, Sanabria JR, Strasberg SM (1992) Proposed classification of complications of surgery with examples of utility in cholecystectomy. Surgery 111(5):518-526

18. Bassi C, Marchegiani G, Dervenis C et al (2017) The 2016 update of the International Study Group (ISGPS) definition and grading of postoperative pancreatic fistula: 11 years after. Surgery 161(3):584-591

19. Cai J, Ramanathan R, Zenati MS et al (2019) Robotic pancreaticoduodenectomy is associated with decreased clinically relevant pancreatic fistulas: a propensity-matched analysis. J Gastrointest Surg 24(5):1111-1118

20. Ausania F, Landi F, Martinez-Perez A, Fondevila C (2019) A meta-analysis of randomized controlled trials comparing laparoscopic vs open pancreaticoduodenectomy. HPB 21(12):1613-1620

21. Nickel F, Haney CM, Kowalewski KF et al (2019) Laparoscopic versus open pancreaticoduodenectomy: a systematic review and meta-analysis of randomized controlled trials. Ann Surg 271(1):54-66

22. Nassour I, Wang SC, Christie A et al (2018) Minimally invasive versus open pancreaticoduodenectomy: a propensitymatched study from a national cohort of patients. Ann Surg 268(1):151-157

23. Lonjon G, Boutron I, Trinquart L et al (2014) Comparison of treatment effect estimates from prospective nonrandomized studies with propensity score analysis and randomized controlled trials of surgical procedures. Ann Surg 259(1):18-25

24. Williams GA, Liu J, Chapman WC et al (2020) Composite length of stay, an outcome measure of postoperative and readmission length of stays in pancreatoduodenectomy. J Gastrointest Surg 24(9):2062-2069

Publisher's Note Springer Nature remains neutral with regard to jurisdictional claims in published maps and institutional affiliations. 